\title{
Predicting adverse outcomes in obstetric sepsis using modified sepsis scores
}

\author{
Amita Ray, Debjani Goswami, Bharath Kumar \\ Corresponding author: Dr. Amita Ray, Professor and HOD, Department of Obstetrics and \\ Gynecology, IQ City Medical College Durgapur, West Bengal, India; Email : amitarays@gmail.com
}

Distributed under Attribution-Non Commercial - Share Alike 4.0 International (CC BY-NC-SA 4.0)

\begin{abstract}
Background: Obstetric sepsis continues to be one of the major causes of maternal mortality. Assessment of obstetric sepsis and management plans need to take into consideration the altered immunological and physiological responses of pregnancy. Obstetrically modified Sequential Organ Failure Assessment (omSOFA) and quick omSOFA (omqSOFA) scores have been proposed which take into account these altered responses. Objectives: We did this study with the objective of comparing the omSOFA and omqSOFA scores with the original SOFA and original quick SOFA (q-SOFA) scores to find which of these better predicted the outcomes of mortality, intensive care unit (ICU) stay and hospital stay in patients with obstetric sepsis. Materials and methods: All cases of obstetric sepsis admitted to our hospital ICU during the period August 2018 to July 2019 were included in this study. The SOFA scores, and the obstetrically modified SOFA scores were calculated, sepsis biomarkers send and all were assessed for their ability to predict mortality using binary logistic regression. Results: There was a significant association $(p<0.001)$ between mortality and the SOFA, om-SOFA and omq-SOFA scores. The q-SOFA score was not significantly associated with mortality ( $\mathrm{p}$ value $=0.315$ ). The om-SOFA proved to be the best predictor of mortality. Adding biomarkers to the calculations did not significantly improve the predictability of the om-SOFA score. Conclusion: Modifying the SOFA scores has increased the predictability of mortality in obstetric sepsis and these should be used when assessing and monitoring this special category of sepsis patients.
\end{abstract}

Keywords: Sepsis, Sequential Organ Failure Assessment (SOFA), mortality.

Sepsis continues to be one of the major causes of maternal mortality, ${ }^{1}$. According to some estimatesthe prevalence of puerperal sepsis alone is $4.4 \%$. When all infective pathologies are combined it may contribute up to $25-40 \%$ of maternal mortality ${ }^{1}$. Using an expert consensus processthe European Society of Intensive Care Medicine and the Society of Critical Care Medicine defined sepsis as a life threatening organ dysfunction secondary to a dysregulated response to infection. According to SEPSIS it is this organ dysfunction which differentiates infection and systemic inflammatory response from sepsis, including obstetric sepsis. ${ }^{3}$ To clinically assess organ dysfunction (and thereby sepsis) a Sequential Organ Failure Assessment score (SOFA) (Appendix-1) was developed wherein a score of more than 2 was a predictor of $10 \%$ mortality. Since the assessment of the SOFA score included laboratory parameters a quick SOFA score (qSOFA score) (Appendix1) score was developed which had the advantage of rapid clinical assessment without having to wait for laboratory results ${ }^{3}$.It is to be noted that the SOFA scores do not give a cut off for sepsis but a score of more than 2 predicts a higher mortality rate $(10 \%)$.

Received: $18^{\text {th }}$ May 2020, Peer review completed: $24^{\text {th }}$ June 2020, Accepted: $27^{\text {th }}$ June 2020.

Ray A, Goswami D, Kumar B. Predicting adverse outcomes in obstetric sepsis using modified sepsis scores. The New Indian Journal of OBGYN. 2021; 7(2): 129-34. 
Since physiological and immunological responses in pregnancy are altered, and pregnancy has the fetus as an added organ, the Society of Obstetric Medicine Australia and New Zealand suggested an obstetrically modified SOFA (omSOFA) (Appendix 2)and an obstetrically modified qSOFA (omqSOFA) (Appendix 2) score for better risk stratification and management of obstetric sepsis ${ }^{2}$.

We did this study with the Appendix1: Original scores [Sequential Organ Failure Assessment Score (SOFA Score)]

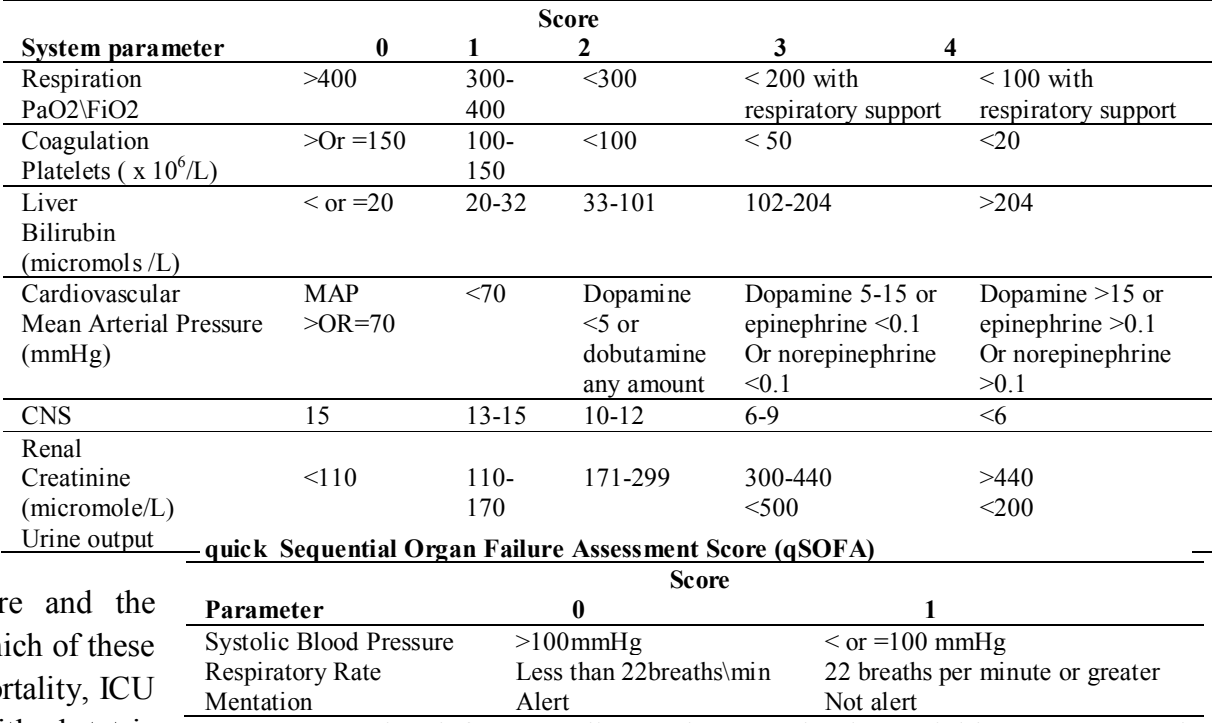
objective of comparing the obstetrically modified SOFA score and the original SOFA scores to find out which of these better predicted the outcomes of mortality, ICU stay and hospital stay in patients with obstetric level for mortality. Subsequently the variables were passed sepsis in our hospital. This would improve quality and efficiency of care, help in patient counseling, guide adequate resource use particularly in low resource settings and could also be used as a template in hospital obstetric units.

\section{Materials and methods}

This study was conducted at a tertiary care hospital. Ethical approval was taken from the Institutional Ethical Committee and all obstetric sepsis cases reporting to the hospital during the period of August 2018 to July 2019 were included. All pregnant women irrespective of the period of gestation and all women within 42 days of delivery or abortion with sepsis were included in the study. To define sepsis clinically, at the time of admission, we used a score of more than two in both the q SOFA and the omq SOFA scores (Appendices 1\& 2). The investigations done were blood counts, blood cultures, other appropriate cultures (eg high vaginal swab, wound swab),renal and liver functions and coagulation parameters. Biomarkers of sepsis CRP, serumlactate, pro-calcitonin, a blood gas analysis and a CTG INST for the fetus were also done. Based on the clinical and the laboratory parameters, SOFA and omSOFA, scores were calculated for each of these women (Appendices 1\&2). The patients were followed upto the day of discharge or death. The outcome measures studied were in house mortality, ICU stay and hospital stay. Each of the scores (SOFA, q SOFA, omSOFA, omqSOFA) were assessed for their predictability in terms of these outcome measures. The variables chosen for the model was based on chi square method with $0.1 \%$ through logistical regression to obtain adjusted $\mathrm{p}$ - values.

Appendix 2: Obstetrically modified scores [Obstetrically Modified Sequential Organ Failure Score (omSOFA)]

\begin{tabular}{|c|c|c|c|}
\hline \multirow[t]{2}{*}{ System parameters } & \multicolumn{2}{|r|}{ Score } & \\
\hline & $\mathbf{0}$ & 1 & 2 \\
\hline Respiration: $\mathrm{PaO} 2 \backslash \mathrm{FiO} 2$ & $>400$ & $300-400$ & $<300$ \\
\hline $\begin{array}{l}\text { Coagulation: Platelets } \\
\left(\times 10^{6} / \mathrm{L}\right)\end{array}$ & $>$ Or $=150$ & $100-150$ & $<100$ \\
\hline $\begin{array}{l}\text { Liver: Bilirubin } \\
\text { (micromols/L) }\end{array}$ & $<$ or $=20$ & $20-32$ & $>32$ \\
\hline $\begin{array}{l}\text { Cardiovascular: Mean } \\
\text { Arterial Pressure }(\mathrm{mmHg})\end{array}$ & $\begin{array}{l}\text { MAP } \\
>\mathrm{OR}=70\end{array}$ & $<70$ & $\begin{array}{l}\text { Vassopressors } \\
\text { required }\end{array}$ \\
\hline CNS & Alert & $\begin{array}{l}\text { Rousable by } \\
\text { voice }\end{array}$ & $\begin{array}{l}\text { Rousable by } \\
\text { pain }\end{array}$ \\
\hline $\begin{array}{l}\text { Renal: Creatinine } \\
\text { (micromole/L) }\end{array}$ & or $=90$ & $90-120$ & $>120$ \\
\hline
\end{tabular}

Obstetrically Modified Quick SOFA Score (omqSOFA)

\begin{tabular}{lll}
\hline Parameters & \multicolumn{1}{c}{ 0 Score } & \multicolumn{1}{c}{} \\
\hline $\begin{array}{l}\text { Systolic Blood } \\
\text { Pressure }\end{array}$ & $>$ or $=90 \mathrm{mmHg}$ & $<90 \mathrm{mmHg}$ \\
\hline Respiratory Rate & Less than 25breaths $\backslash$ min & $\begin{array}{l}25 \text { breaths per minute } \\
\text { or greater }\end{array}$ \\
\hline Altered mentation & Alert & Not alert \\
\hline
\end{tabular}

\section{Results}

Average age of the study population was 31 years and majority of them were in the age group 30-39yrs (table 1). Most the ICU admissions were for non-obstetric causes of sepsis, the most common being respiratory tract infections. Puerperal sepsis was the most frequent cause of obstetric sepsis (table 2). 
The New Indian Journal of OBGYN. 2021 (January-June);7(2)

Table 1: General characteristics of our population

\begin{tabular}{llc}
\hline S No & Age & Number (Percentage) \\
\hline 1 & $<20$ & $8(10 \%)$ \\
2 & $20-29$ & $24(30 \%)$ \\
3 & $30-39$ & $32(40 \%)$ \\
4 & $>39$ & $12(20 \%)$ \\
\hline \multicolumn{2}{c}{ Respiratory system was involved in majority of the }
\end{tabular}

Respiratory system was involved in majority of the women followed by the cardiovascular and renal system (table 3). This was on the day of admission to the ICU when we calculated the scores. In woman who did not survive more organ systems were involved with the passage of time.

Table 2: Causes of sepsis

\begin{tabular}{lll}
\hline Parameters & Antepartum & Postpartum \\
\hline Obstetric & Septic abortion $\mathrm{n}=6$ & Puerperal sepsis $\mathrm{n}=14$ \\
cause $\mathrm{n}=30$ & Chorioamnionitis $\mathrm{n}=9$ & Wound infection $\mathrm{n}=1$ \\
\hline Non obstetric & Respiratory $\mathrm{n}=20$ & Respiratory $\mathrm{n}=8$ \\
causes $\mathrm{n}=48$ & UTI $\mathrm{n}=2$ & UTI $\mathrm{n}=10$ \\
& Others $\mathrm{n}=3$ & Others $\mathrm{n}=5$ \\
\hline Total 78 & $\mathrm{~N}=40$ & $\mathrm{~N}=38$ \\
\hline
\end{tabular}

Using Fisher's exact test we found a significant association $(p<0.001)$ between mortality and the SOFA, omSOFA and omqSOFA scores. The qSOFA score was not significantly associated with mortality ( $p$ value 0.315 ) (table 4).

Table 3: Organ systems involved (numbers and percentages)

\begin{tabular}{lc} 
Organ system involved $^{*}$ & Number (Percentage) $^{*}$ \\
\hline Respiratory system & $28(36 \%)$ \\
CVS & $20(26 \%)$ \\
Liver & $19(24 \%)$ \\
Renal & $11(14 \%)$ \\
Coagulation & $11(14 \%)$ \\
CNS & $2(3 \%)$ \\
\hline
\end{tabular}

Using binary regression model among the scores that were significantly associated with mortality omSOFA proved to be the best predictor of mortality (table 5). Among all the scores studied omSOFA was the best predictor based

Table 4: Association of scores with mortality

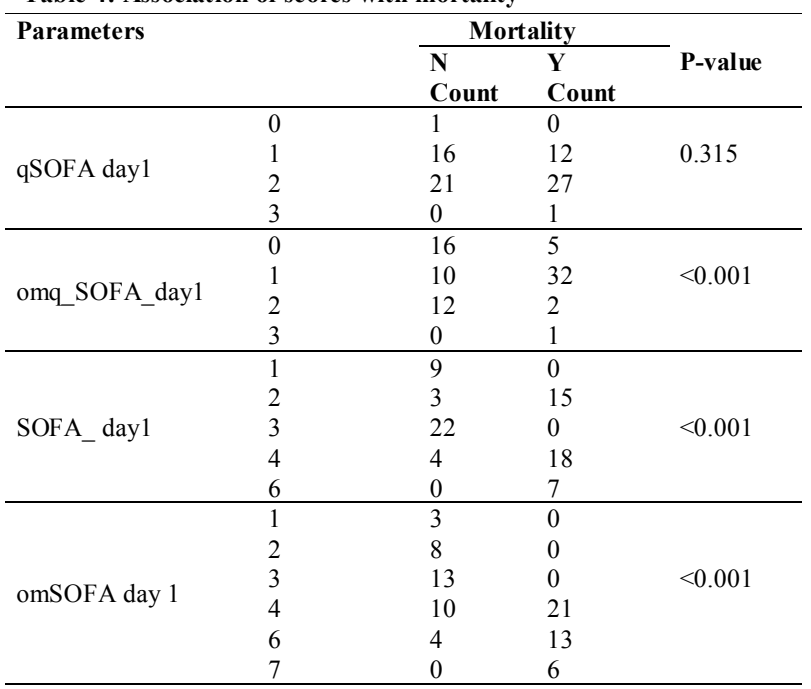

on the above model with p-value less than 0.001 .

Table 5: Group Associations to identify the best predictor (sepsis scores) of mortality

\begin{tabular}{lllll} 
Parameters & $\begin{array}{l}\text { Odds } \\
\text { Ratio }\end{array}$ & $\begin{array}{l}\text { 95\% CI for Odds } \\
\text { Ratio }\end{array}$ & P-value \\
\cline { 3 - 4 } & & Lower & Upper & \\
\hline q_SOFA_day1 & 2.062 & 0.429 & 9.924 & 0.366 \\
omq_SOFA_day1 & 0.899 & 0.276 & 2.931 & 0.860 \\
SOFA_day1 & 0.706 & 0.326 & 1.527 & 0.376 \\
omSOFA day1 & 3.915 & 1.904 & 8.053 & $<0.001$ \\
\hline
\end{tabular}

The analysis method was binary logistic regression considering the above scores are continuous.

Multivariable binary logistic regression was done to check the association of mortality with increased levels of serum lactate and pro-calcitonin. The adjusted odds ratio suggest that with increased levels of serum lactate, mortality is 4.794 ( CI 2.281, 10.079) times greater with a p-value of $<0.001$ whereas with increased levels of pro-calcitonin mortality is 1.137 (CI $1.038,1.1246)$ times greater with a p-value of 0.006 (table 6). Thus both serum lactate and procalcitonin are significantly associated with mortality but serum lactate performs better.

Table 6: Association of mortality with sepsis markers (serum lactate and pro-calcitonin)

\begin{tabular}{lllll}
\hline Parameters & $\begin{array}{l}\text { Odds } \\
\text { Ratio }\end{array}$ & \multicolumn{2}{c}{$\mathbf{9 5 \%}$ CI } & \multicolumn{2}{c}{ P-value } \\
\cline { 3 - 4 } & Lower & Upper & \\
\hline Serum lactate & 4.794 & 2.281 & 10.079 & 0.000 \\
Pro-calcitonin & 1.137 & 1.038 & 1.246 & 0.006 \\
\hline
\end{tabular}

To see whether combining the best score (om SOFA) with the biomarkers, increases the predictability as regards to mortality we included the scores and the biomarkers of sepsis namely the pro-calcitonin and serum lactate in the logistic regression model. Best fit was identified using $\mathrm{R}$ square value (the variability explained by the model): greater the $\mathrm{R}$ square value greater would be the predictability. The results showed that $R$ square was maximum when all the three were combined that is omSOFA, procalcitonin and serum lactate. However when compared to the $\mathrm{R}$ square value of the score, the $\mathrm{R}$ square value of the scores plus the biomarkers did not show significant improvement leading to the conclusion that the score in themselves were adequately capable of predicting the mortality outcomes (table 7).

Table 7: Association of mortality with combinations of serum markers and sepsis scores

\begin{tabular}{lllll}
\hline Parameters & $\begin{array}{l}\text { Odds } \\
\text { ratio }\end{array}$ & \multicolumn{2}{l}{$\mathbf{9 5 \%}$ CI } & P value \\
\cline { 3 - 4 } & Lower & Upper & \\
\hline Serum lactate & 15.753 & 1.283 & 193.479 & .031 \\
Procalcitonin & 1.301 & 1.018 & 1.664 & .036 \\
omSOFA & 10.131 & 1.113 & 92.239 & .040 \\
\hline
\end{tabular}

When considering the ICU stay and hospital stay both q SOFA and omqSOFA were significantly correlated with each other. The SOFA and omSOFA did not show correlation probably because higher these scores higher was the mortality in the shortest time from admission and thus 
there would be paradoxically lesser days of stay in the ICU (table 8 \& 9).

Table 8: Correlation of scores with number of days in the ICU

\begin{tabular}{llll}
\hline Parameters & N & Correlation & p-value \\
\hline q_SOFA_1 & 38 & -0.781 & $<0.001$ \\
omq_SOFA_1 & 38 & -0.694 & $<0.001$ \\
SOFA_1 & 38 & -0.183 & 0.2708 \\
omSOFA_1 & 38 & -0.027 & 0.8706 \\
\hline \multicolumn{4}{l}{ Spearman rank correlation was } \\
number to assess the correlation of \\
nof sepsis)
\end{tabular}

Table 9: Correlation of scores with the number of days in the hospital

\begin{tabular}{llll}
\hline Parameters & N & Correlation & p-value \\
\hline q_SOFA_1 & 78 & -0.199 & 0.0806 \\
omq_SOFA_1 & 78 & -0.271 & 0.0162 \\
SOFA_1 & 78 & -0.585 & $<0.001$ \\
omSOFA_ 1 & 78 & -0.656 & $<0.001$ \\
\hline \multicolumn{4}{l}{ Spearman rank correlation was used to assess the correlation of } \\
number of days in hospital stay with the covariates (the scores \\
and biomarkers of sepsis )
\end{tabular}

\section{Discussion}

The third international consensus on sepsis has defined sepsis as a life threatening organ dysfunction due to dysregulated response to infection. It has also given the SOFA score which does not give a cut off value to confirm or decline sepsis; rather it gives a prognostic indicator of the ominous outcomes. A SOFA score above 2 predicts a mortality of $10 \%$. This consensus was given based on large data bases. However it excluded pregnancy, which is a unique condition because of the associated physiological and immunological changes and the presence of the fetus which could be thought of as another organ. ${ }^{3}$ It is because of these changes in pregnancy that the SOFA score would not be as accurate when predicting mortality outcomes in maternal sepsis. $^{2}$

Modifications of the SOFA score ${ }^{2}$ have been made since then to address this issue as well as issues of resource constrained settings where all lab parameters required to calculate the SOFA score would not be possible at once ${ }^{4-6}$. Earlier to the Sepsis consensus of 2016, scores which were particularly suited to the obstetric patient and maternal sepsis have also been developed ${ }^{7}$.

The SOMANZ (Society of Obstetric Medicine Australia and New Zealand) has provided a obstetrically modified SOFA (omSOFA) score which has taken into account the physiological changes in pregnancy ${ }^{3}$. Besides the omSOFA this guideline also gives an obstetrically modified quick SOFA(omqSOFA) score for quick assessment and triaging . The purpose of our study was to find whether "obstetrically" modifying these score improves their prognostic ability for adverse outcomes when compared to the original scores in women with obstetric or maternal sepsis.

Results of our study showed that SOFA, omSOFA and omqSOFA were significantly associated with mortality and all three thus could be used as good prognostic markers for adverse outcomes in maternal sepsis as well as to guide management. Among the three the omSOFA score performs better than the other two. The qSOFA however does not show a significant association with maternal mortality. According to this study it would appear that modifying the score has improved its performance in cases of obstetric sepsis. More importantly the omqSOFA performs significantly better than the qSOFA and would be very useful both for evaluating, triaging, guiding management as well as counseling as soon as a case of maternal sepsis arrives to the hospital without having to wait for investigation results. On review of literature there were several studies using modified scores for triaging and prognostication $^{4-8}$. Most of these studies had modified the SOFA scores by substituting clinical assessment scores in lieu of the laboratory values ${ }^{5-8}$. The aim was to reduce the costs and save the time that would be needed to get the results before an assessment could be made. Most of these studies found that modifying the scores did not significantly alter their predictability when compared to the original scores. However most of these studies exclude pregnant women and the studies were done in a single centre with a relatively small number of participants ${ }^{5-7}$.

We found only one study which specifically addressed obstetric \maternal sepsis and used modified SOFA scores to predict outcomes ${ }^{8}$. The study came to the conclusion that the modified scores performed significantly well when predicting outcomes . This study however included pregnant women with preeclampsia, eclampsia and other conditions associated with pregnancy which lead to multi-organ failure and was therefore not limited to "sepsis" and was not specific to dysregulated organ response to infection alone.

We also included some biomarkers in our study to see whether they by themselves or in combination with sepsis scores enhanced the prognostic and monitoring efficacy in maternal sepsis. Sepsis biomarkers have been used since long for diagnosing, monitoring and planning management for patients in sepsis and have also been the subject of numerous research studies ${ }^{9-12}$. The commonly used biomarkers are white blood cell count (WBC), C-reactive protein (CRP), pro-calcitonin (PCT) and serum lactate. White blood cell count and C-Reactive protein are found to 
The New Indian Journal of OBGYN. 2021 (January-June);7(2)

be elevated in conditions other than sepsis. Pro-calcitonin on the other hand is more specific for bacterial infections ${ }^{9}$. Lactate is another marker commonly used in sepsis. Studies trying to ascertain which among the two (procalcitonin and serum lactate) is a better marker of sepsis have given conflicting results. ${ }^{9-12}$

Due to the physiological and immunological changes in pregnancy the use of these biomarkers in obstetric sepsis cannot be extrapolated from the general population. WBC and CRP are physiologically elevated in pregnancy and normal levels of pro-calcitonin in pregnancy are not well defined. ${ }^{9}$ Lactate although more consistent in pregnancy than all the other three may be increased as a result of the increased metabolic load of pregnancy ${ }^{9-11}$. Despite this and among all the four arise in lactate levels is a good indicator of tissue hypo-perfusion and hypoxia which is the end common pathway of sepsis. CRP and WBC are more of inflammatory markers. ${ }^{10-12}$

When taking into consideration the biomarkers of sepsis our study concluded that increased levels of both serum lactate and procalcitonin were significantly associated with mortality. This has been the finding in other studies as well. ${ }^{9-12}$ Combining the best predictability score that is the omSOFA with these biomarkers did improve the predictability but not to a significant extent. This leads us to believe that even without investigating for these biomarkers omqSOFA score is a good triaging tool for cases of maternal sepsis. Further on obtaining the investigation results the omSOFA performs as a better prognostic and monitoring tool as compared to the original SOFA score.

Strengths and limitation: Unlike other studies which have considered adverse outcomes in all obstetric critical care cases this study has specifically outlined its findings for cases of maternal sepsis using the obstetrically modified and validated sepsis scores. This study has the limitations of being a single centric study with a small number of patients. Predictability may have been affected by this.

\section{Conclusion}

From the results of this study it can be concluded that modifying the SOFA scores has increased the relevance and the predictability of mortality in this special category of patients with sepsis. Thus when managing women with obstetric sepsis the obstetrically modified sepsis scores should be used. Among the serum biomarkers serum lactate correlates significantly with mortality in maternal sepsis but when combined with the obstetrically modified SOFA does not increase predictability for adverse outcomes to a significant degree.

\section{Conflict of interest: None. Disclaimer: Nil.}

\section{References}

1. Greer O, Shah NM, Johnson MR. Maternal sepsis update: current management and controversies. The Obstetrician \& Gynaecologist. 2020; 22:45-55.

2. Bowyer L, Robinson HL, Barrett H, Crozier TM, Giles $\mathrm{M}$,Idel I, et al. SOMANZ guidelines for the investigation andmanagement sepsis in pregnancy. Aust N Z J Obstet Gynaecol. 2017; 57: 540-51

3. Seymour CW, Liu VX, Iwashyna TJ, Brunkhorst FM, Rea TD, Scherag A, et al. Assessment of clinical criteria for sepsis For the Third International Consensus Definitions for Sepsis and Septic Shock. JAMA. 2016;315(8):762-74

4. Bonet M, Souza JP, Abalos E, Fawole B, Knight M, et al. The global maternal sepsis study and awareness campaign (GLOSS): study protocol. Reproductive Health. 201.8 15(1):16

5. Gholipour Baradari A, Sharifi H, Firouzian A, Daneshiyan M, Aarabi M, Talebiyan Kiakolaye Y, et al. Comparison of Proposed Modified and Original Sequential Organ Failure Assessment Scores in Predicting ICU Mortality: A Prospective, Observational, Follow-Up Study. Scientifica (Cairo). 2016; 2016: 7379325.

6. Grissom CK, Brown SM, Kuttler KG, Boltax JP, Jones $\mathrm{J}$, Jephson AR, et al. A modified sequential organ failure assessment score for critical care triage. Dis Med Publ Health Prep. 2010; 4(4):277-284.

7. Albright CM, Ali TN, Lopes V, Rouse DJ, Anderson BL. The Sepsis in Obstetrics Score: a model to identify risk of morbidity from sepsis in pregnancy. Am J ObstetGynecol. 2014; 211: 39.e1-8.

8. Esquivel LAB, Urbina JM, Zerón HM. Approach to an obstetric prognosis scale: The modified SOFA scale. Ghana Med J. 2016; 50(3): 129-35

9. Nargis W, Ibrahim Md, Ahamed BU. Procalcitonin versus C-reactive protein: Usefulness as biomarker of sepsis in ICU patients. Int J Crit Illn Inj Sci. 2014 ; 4(3): 195-9

10. Lin CT, Lu JJ, Chen YC, Kok VC, Horng JT. Diagnostic value of serum procalcitonin, lactate, and high-sensitivity C-reactive protein for predicting 
The New Indian Journal of OBGYN. 2021 (January-June);7(2)

bacteremia in adult patients in the emergency department. Peer J. 2017; 5: e4094

11. Shetty AL, Thompson K, Byth K, Macaskill P, Green M, Fullick M, et al. Serum lactate cut-offs as a risk stratification tool for in-hospital adverse outcomes in emergency department patients screened for suspected sepsis. BMJ Open. 2018; 8: e015492

12. Faix JD. Biomarkers of sepsis. Crit Rev Clin Lab Sci. 2013; 50(1): 23-3 